'Escuela de Psicología, Universidad Católica del Norte. Antofagasta, Chile.

2Escuela de Psicología, Universidad del Bío Bío. Chillán, Chile.

${ }^{3}$ Departamento de Psicología, Universidad Católica del Maule. Talca, Chile.

"Escuela de Psicología, Universidad Alberto Hurtado. aPsicóloga, Doctora en Psicología.

bPsicólogo, Mg. en Psicología Social.

CPsicóloga, Mg. en Psicología Clínica.

¿Psicólogo, Doctor en Psicología Social.

ePsicólogo, Doctor en Psicología Clínica y de la Salud.

Esta investigación ha sido financiada con la ayuda para proyectos de investigación concedida por el Fondo Nacional de Desarrollo Científico y Tecnológico del Gobierno de Chile FONDECYT 11100141, FONDECYT 1110887. Los autores declaran no tener conflictos de interés.

Recibido el 16 de abril de 2019, aceptado el 28 de enero de 2020.

Correspondencia a: Mónica Guzmán-González Avenida Angamos 0610, Antofagasta, Chile. moguzman@ucn.c

\section{Propuesta de valores de referencia para la Escala de Dificultades de Regulación Emocional (DERS-E) en población adulta chilena}

\author{
MÓNICA GUZMÁN-GONZÁLEZ,a, RODOLFO MENDOZA-LLANOS ${ }^{2, \mathrm{~b}}$, \\ LUSMENIA GARRIDO-ROJAS ${ }^{3, \mathrm{c}}$, JAIME BARRIENTOS ${ }^{4, \mathrm{~d}}$, \\ ALFONSO URZÚA ${ }^{1, \mathrm{e}}$
}

\section{Cut-off points of the difficulties in Emotion Regulation Scale for the Chilean population}

Background: There are no reference values for the Difficulties in Emotion Regulation Scale (DERS-E) for the Chilean population. Aim: To elaborate reference values for the interpretation of the DERS-E for the Chilean population. Material and Methods: The Difficulties in Emotion Regulation Scale was answered by participants from the general population (1,153 heterosexual men and women and 467 gay and lesbian individuals) and a group of 351 participants with emotional complaints. Receiver operating characteristic (ROC) curves were used to obtain the reference values. A concordance index was determined, and the odds ratio was calculated to evaluate the probability of having mental health problems comparing participants with emotional complaints with those from the general population. Results: The DERS-E cut-off score was 73 points for the Chilean population. No differences were detected for emotional regulation difficulties between men and women or by sexual orientation. Age had a weak association with emotional regulation difficulties. Conclusions: DERS-E has an adequate diagnostic capacity, allowing to differentiate between those who have high or low emotional regulation difficulties.

(Rev Med Chile 2020; 148: 644-652)

Key words: Emotional Regulation; Emotions; Reference Values.
L os trastornos de salud mental representan problemas de salud pública ${ }^{1}$ que tienen un alto costo a nivel individual, social y sanitario $^{2}$. En Chile, los problemas de salud mental constituyen la principal fuente de carga de enfermedad ${ }^{3}$, por lo que la mejora en estrategias de abordaje y diagnóstico precoz en distintas poblaciones son contribuciones permanentes al sistema de salud pública. Al respecto, se ha concluido que la regulación emocional tiene un valor significativo en la investigación en salud mental, dado que aparece como un factor relevante en el desarrollo, mantenimiento y tratamiento de distintos desórdenes mentales ${ }^{4}$.

La regulación emocional puede ser definida como un conjunto de procesos extrínsecos e intrínsecos responsables de monitorear, evaluar y modificar las reacciones emocionales, especialmente su intensidad y características en el tiempo, con el fin de alcanzar las propias metas.

Gratz y Roemer ${ }^{6}$ proponen que una regulación emocional adaptativa implica tener conciencia, comprensión y aceptación de las emociones, así como la capacidad de controlar las conductas 
impulsivas y actuar de acuerdo con las metas deseadas cuando se experimentan emociones negativas.

En congruencia con esta conceptualización, Gratz y Roemer ${ }^{6}$ delimitaron una propuesta de las dificultades de regulación emocional (DRE), considerando las siguientes dimensiones: descontrol emocional, que alude a la dificultad para mantener el control del comportamiento cuando se experimentan emociones negativas; interferencia cotidiana, referida a la dificultad para concentrarse y cumplir tareas cuando se experimentan emociones negativas; desatención emocional, entendida como la dificultad para atender y tener conocimiento de las emociones; confusión emocional, que refiere a la dificultad para conocer y tener claridad acerca de las emociones que se están experimentando; y el rechazo emocional que incluye reacciones emocionales negativas en respuesta a una emoción negativa o de no aceptación del propio malestar.

En la actualidad existe amplia evidencia acerca del rol que juegan las DRE en el surgimiento y mantención de diversos problemas de salud física y mental ${ }^{7}$. Las DRE se han asociado a problemas de salud física (hipertensión arterial ${ }^{8}$, problemas cardíacos $^{9}$ y cáncer ${ }^{10}$ ) y problemas de salud mental (depresión ${ }^{11-13}$, trastornos de externalización e internalización ${ }^{14}$, trastornos de personalidad ${ }^{15}$, esquizofrenia ${ }^{16}$, trastornos de ansiedad ${ }^{17}$, trastorno obsesivo-compulsivo ${ }^{18}$, ataques de pánico $^{19}$, trastorno de acumulación ${ }^{20}$, síntomas de estrés postraumático $^{21}$, autolesiones ${ }^{22}$, desórdenes alimentarios $^{23}$ y consumo de sustancias ${ }^{24,25}$ ). Tiene particular importancia que antecedentes internacionales revelan que dentro de los problemas de salud mental, la depresión y síntomas depresivos tendrían una prevalencia de hasta $20 \%$ de la población ${ }^{12}$, y su curso o aparición tiene una fuerte asociación con la desregulación emocional ${ }^{1-13}$.

Por otro lado, también existe evidencia de que, a raíz de la exposición a situaciones de estrés minoritario, las DRE serían mayores en grupos de personas gay y lesbianas (GL) que en personas heterosexuales ${ }^{26-29}$.

A nivel mundial existen, a lo menos, 15 instrumentos que permiten medir la regulación emocional en la adultez ${ }^{30}$ y uno de los más utilizados para evaluar las DRE es la Escala de Dificultades de Regulación Emocional (Difficulties in Emotion Regulation Scale) (DERS) ${ }^{6}$, por ser un instrumento que permite capturar el carácter multidimensional de este constructo, desde una conceptualización integradora del mismo. A la fecha, se cuenta con adaptaciones y validaciones de la DERS en distintos países del mundo ${ }^{30-38}$, incluyendo Chile ${ }^{30}$; sin embargo, y para el contexto chileno, no se dispone hasta ahora de normas de interpretación que permitan su uso como medida de tamizaje o como referencia para la toma de decisiones en contextos clínicos. Las buenas propiedades psicométricas de la versión chilena del DERS ${ }^{30}$ que justifican su utilización, se refrendan a partir de un estudio en que, al evaluar el rigor psicométrico de diversas escalas de regulación emocional ocupadas en población hispanoparlante (DERS-E), esta versión ocupó el tercer lugar de un total de 34 instrumentos revisados ${ }^{39}$.

Con base en estos antecedentes, el presente trabajo se propuso los siguientes objetivos: (a) elaborar normas de referencia para la DERS-E; (b) evaluar diferencias en las DRE en función del sexo, orientación sexual y edad; (c) evaluar la sensibilidad del punto de corte en población general chilena y (d) explorar un punto de corte óptimo para diferenciar entre personas con bajas y elevadas DRE, y entre personas con y sin malestar socioemocional clínicamente significativo.

\section{Material y Método}

\section{Participantes y procedimiento}

Se ocuparon muestras no probabilísticas de tres estudios independientes que abarcaron participantes mayores de 18 años, chilenos y chilenas de la zona norte $(66,62 \%)$, centro $(26,33 \%)$ y sur $(7,05 \%)$ del país.

Una muestra de población heterosexual ( $\mathrm{n}=1.153 ; 50,56 \%$ mujeres), una muestra de personas gay y lesbianas $(\mathrm{n}=467 ; 42,61 \%$ mujeres $)$ y una muestra clínica, conformada por personas que declaraban malestar socioemocional clínicamente significativo ( $\mathrm{n}=351 ; 58,69 \%$ de mujeres).

La comparación de las primeras dos muestras responde a la necesidad ética de selección equitativa de individuos ${ }^{40,41}$, evitando la heteronorma en la elaboración de parámetros y la subrepresentación de grupos minoritarios expuestos a factores de riesgo anclados en lo social, como la discriminación y el prejuicio, que tienen repercusiones sobre el bienestar y la salud mental ${ }^{42,43}$.

En todos los casos, la recolección de datos se 
realizó a través de cuestionarios de autorreporte, respondidos en forma individual bajo la modalidad de lápiz y papel.

El estudio contó con la aprobación de los comités de ética de la Universidad Católica del Norte y de la Universidad de Santiago y se desarrolló siguiendo los principios establecidos en la declaración de Helsinki. En todos los casos, los participantes fueron informados acerca de los objetivos del estudio y firmaron un consentimiento informado.

\section{Instrumentos}

Escala de Dificultades de Regulación Emocional. Este es un cuestionario de autorreporte creado por Gratz y Roemer ${ }^{6}$, cuyo objetivo es evaluar problemas de regulación emocional. Para este estudio se utilizó la versión validada en Chile, DERS-E ${ }^{30}$, compuesta por 25 ítems en formato Likert de 5 puntos ( 1 = Casi nunca, 5 = Casi siempre), donde puntajes más altos indican mayores dificultades en la regulación emocional. La versión chilena contiene cinco subescalas (rechazo emocional, descontrol emocional, interferencia emocional, desatención emocional y confusión emocional), y posee buenas propiedades psicométricas en términos de su validez de constructo como de sus índices de confiabilidad, con valores fluctuantes entre $\alpha=0,71$ y $\alpha=0,89$ y una fiabilidad total de $\alpha=0,92^{34}$.

Cuestionario para evaluación de resultados y evolución en psicoterapia (Outcome Questionnaire [OQ-45.2]). Esta escala fue diseñada para la evaluación de los resultados en psicoterapia ${ }^{44}$, no obstante, también mide ajuste socioemocional y fue validada en Chile ${ }^{45}$. Se compone de 45 reactivos distribuidos en tres ámbitos de malestar socioemocional: sintomatología ansioso-depresiva, relaciones interpersonales y rol social. Su formato de respuesta es tipo Likert, de cinco puntos $(0=\mathrm{Nunca}$, $4=$ Casi siempre) en función de lo experimentado en los últimos 7 días, y entrega un puntaje total que va de 0 a 180 puntos, en el que puntajes más altos indican un mayor malestar emocional, con un punto de corte de $73^{45}$. Para este estudio, la confiabilidad de la escala es $\alpha=0,92 ; \omega=0,92$.

Escala de Depresión del Centro para Estudios Epidemiológicos (The Center for Epidemiologic
Studies Depression Scale [CES-D]). Escala de autorreporte del Centro para Estudios Epidemiológicos de Estados Unidos de Norteamérica, validada en Chile y con adecuadas propiedades psicométricas ${ }^{46}$. Consta de 20 ítems que describen los síntomas más habituales y representativos del trastorno depresivo, donde las personas deben indicar la frecuencia con la que se experimentó cada síntoma durante la semana anterior, utilizando una escala de cuatro alternativas que van desde "Rara vez o ninguna vez (1 día o menos)", hasta "La mayor parte del tiempo (5 a 7 dias)". Este instrumento establece un punto de corte de $24 / 25$ para identificar a personas con depresión ${ }^{46}$. Para este estudio, la confiabilidad de la escala es $\alpha=0,92, \omega=0,92$.

\section{Análisis Estadístico}

Primero, con las muestras de población general (personas heterosexuales y personas gay y lesbianas [GL]), y con el objetivo de evaluar si era necesario calcular puntajes diferenciados, se hicieron comparaciones entre grupos de acuerdo a sexo y orientación sexual, además de realizar un análisis correlacional y de regresión entre la edad y el DERS-E.

En segundo lugar, para estimar el punto de corte, y dado que el DERS-E permite medir dificultades de regulación emocional ${ }^{12}$ con puntajes que van desde 25 a 125 puntos, con una media teórica de 75 puntos, se define que puntajes sobre 75 en la escala serán considerados "altos" o "con dificultades de regulación emocional" y aquellos menores a 75 serán considerados "bajos" o "sin dificultades de regulación emocional". Con ello se calculó el área bajo la curva con una variable criterio (OQ-45.2) y se elaboró un puntaje de corte, mediante el cálculo de curvas COR.

Luego, y para corroborar la capacidad discriminatoria (sensibilidad y especificidad), se contrastó - para ambos cálculos - la concordancia entre la DERS-E y el OQ-45.2, y el CES-D, a través del índice de Kappa, el cual se interpreta como Leve $(\kappa=0,01-0,20)$, Aceptable $(\kappa=0,21$ $0,40)$, Moderada $(\kappa=0,41-0,60)$, Considerable $(\kappa=0,61-0,80)$ y Casi perfecta $(\kappa=0,81-1,0) 47$. Para efectos de nuestro análisis, se espera que la máxima convergencia entre los instrumentos criterio (OQ-45.2 y CES-D) con DERS-E permitan corroborar el valor de corte. 


\section{Resultados}

\section{Propiedades psicométricas de la escala}

La confiabilidad del instrumento fue adecuada, tanto para su versión global $(\alpha=0,91 ; \omega=0,92)$, como para sus dimensiones en particular: rechazo emocional $(\alpha=0,91)$, descontrol emocional $(\alpha=0,90)$, interferencia emocional $(\alpha=0,89)$, desatención emocional $(\alpha=0,80)$ y confusión emocional $(\alpha=0,75)$.

\section{Cálculo de puntuaciones normativas}

Al comparar los puntajes obtenidos en DERS-E entre hombres $(M=48,29 ; D E=15,38)$ y mujeres $(\mathrm{M}=48,03 ; \mathrm{DE}=15,63)$, no se encontraron diferencias significativas $(t[1626]=-0,34 ; p=0,73$; IC 95\% $[-1,25 ; 1,77], d=0,02)$. Al comparar los puntajes de la DERS-E de acuerdo a orientación sexual (hombres gay, mujeres lesbianas, hombres heterosexuales y mujeres heterosexuales) no se observaron diferencias estadísticamente significativas $\left(\mathrm{F}(3,1624)=0,18 ; p=0,91 ; \eta^{2}=0,001\right)$ entre los grupos. Al observar los estadísticos descriptivos de edad (Tabla 1) y para evaluar si la edad genera diferencias se analizó la correlación entre edad y DERS-E y se encontró una asociación pequeña $(r=-0,05 ; p=0,04)$, y una regresión simple indi- ca que la capacidad explicativa de la varianza de DERS-E que tiene la edad es pequeña $(F(1,1621)=$ 4,$39 ; R_{\text {Adj }}^{2}=0,001 ;$ Beta $\left.=-0,05 ; p=0,04\right)$. Por lo tanto, no se consideró necesario corregir las normas por sexo, orientación sexual ni edad, como se ha hecho en otros estudios previos ${ }^{32}$.

Los estadísticos descriptivos y percentiles de referencia en DERS-E para las distintas muestras evaluadas se pueden observar en la Tabla 2, así como el detalle general de estadísticos por ítems (Tabla 3).

Tabla 1. Composición demográfica de las muestras

\begin{tabular}{|lrrrrr|}
\hline & \multicolumn{5}{c|}{ Edad } \\
& n & Mín Máx Media & DE \\
Población general & & & & & \\
Hombres heterosexuales & 570 & 18 & 65 & 39,8 & 14,3 \\
Mujeres heterosexuales & 583 & 18 & 65 & 39,7 & 13,6 \\
Total & 1.153 & 18 & 65 & 39,7 & 13,2 \\
Hombres gay & 268 & 18 & 67 & 27,7 & 7,6 \\
Mujeres lesbianas & 199 & 18 & 60 & 28,1 & 8,2 \\
Total & 467 & 18 & 67 & 27,9 & 7,9 \\
Muestra clínica & & & & & \\
$\quad$ Hombres & 145 & 19 & 69 & 37,0 & 12,1 \\
Mujeres & 206 & 18 & 68 & 37,8 & 12,1 \\
Total & 351 & 18 & 69 & 37,5 & 12,1 \\
\hline
\end{tabular}

Tabla 2. Estadísticos descriptivos y percentiles de puntaje en DERS-E para las distintas muestras del estudio

\begin{tabular}{|c|c|c|c|}
\hline & $\begin{array}{l}\text { Muestra heterosexual } \\
\qquad(n=1.153)\end{array}$ & $\begin{array}{l}\text { Muestra gays y lesbianas } \\
\qquad(n=467)\end{array}$ & $\begin{array}{l}\text { Muestra clínica } \\
\qquad(n=351)\end{array}$ \\
\hline Media & 48,01 & 48,48 & 70,41 \\
\hline $\mathrm{DE}$ & 15,15 & 16,30 & 17,69 \\
\hline Asimetría & 1,03 & 1,04 & $-0,09$ \\
\hline Curtosis & 0,91 & 0,68 & $-0,02$ \\
\hline Mínimo & 25,00 & 25,00 & 25,00 \\
\hline Máximo & 115,00 & 113,00 & 120,00 \\
\hline P10 & 32,00 & 32,00 & 47,00 \\
\hline P20 & 35,00 & 34,60 & 56,00 \\
\hline P25 & 37,00 & 36,00 & 60,00 \\
\hline P30 & 39,00 & 38,00 & 62,00 \\
\hline P40 & 41,00 & 42,00 & 67,00 \\
\hline P50 & 45,00 & 44,00 & 71,00 \\
\hline P60 & 48,00 & 48,00 & 74,00 \\
\hline P70 & 53,00 & 54,00 & 80,00 \\
\hline P75 & 56,00 & 58,00 & 82,00 \\
\hline P80 & 60,00 & 61,00 & 84,00 \\
\hline P90 & 70,00 & 73,00 & 93,80 \\
\hline
\end{tabular}


Tabla 3. Estadística descriptiva por ítem

\begin{tabular}{|c|c|c|c|c|c|c|}
\hline ítems & M & SD & As & Curt & Mín & Máx \\
\hline 1. Percibo con claridad mis sentimientos & 2,04 & 1,01 & 0,92 & 0,26 & 1 & 5 \\
\hline 2. Presto atención a cómo me siento & 2,17 & 1,07 & 0,74 & $-0,19$ & 1 & 5 \\
\hline 3. Vivo mis emociones como algo desbordante y fuera de control & 2,17 & 1,17 & 0,76 & $-0,35$ & 1 & 5 \\
\hline 4. No tengo ni idea de cómo me siento & 1,76 & 1,06 & 1,43 & 1,29 & 1 & 5 \\
\hline 5. Tengo dificultades para comprender mis sentimientos & 1,96 & 1,13 & 1,08 & 0,32 & 1 & 5 \\
\hline 6. Estoy atento a mis sentimientos & 2,29 & 1,19 & 0,65 & $-0,56$ & 1 & 5 \\
\hline 7. Doy importancia a lo que estoy sintiendo & 2,16 & 1,15 & 0,80 & $-0,26$ & 1 & 5 \\
\hline 8. Estoy confuso sobre lo que siento & 2,05 & 1,14 & 0,97 & 0,08 & 1 & 5 \\
\hline 9. Cuando me encuentro mal, reconozco mis emociones & 2,29 & 1,20 & 0,67 & $-0,57$ & 1 & 5 \\
\hline $\begin{array}{l}\text { 10. Cuando me encuentro mal, me enfado conmigo mismo por sentirme } \\
\text { de esa manera }\end{array}$ & 2,57 & 1,33 & 0,40 & $-1,03$ & 1 & 5 \\
\hline 11. Cuando me encuentro mal, me da vergüenza sentirme de esa manera & 2,09 & 1,26 & 0,93 & $-0,28$ & 1 & 5 \\
\hline 12. Cuando me siento mal, tengo dificultades para completar trabajos & 2,34 & 1,25 & 0,67 & $-0,57$ & 1 & 5 \\
\hline 13. Cuando me encuentro mal, pierdo el control & 1,74 & 1,10 & 1,49 & 1,34 & 1 & 5 \\
\hline 14. Cuando me siento mal, me resulta difícil centrarme en otras cosas & 2,43 & 1,22 & 0,61 & $-0,60$ & 1 & 5 \\
\hline 15. Cuando me encuentro mal, me siento fuera de control & 1,68 & 1,07 & 1,66 & 1,99 & 1 & 5 \\
\hline $\begin{array}{l}\text { 16. Cuando me encuentro mal, me siento avergonzado conmigo mismo } \\
\text { por sentirme de esa manera }\end{array}$ & 1,94 & 1,19 & 1,10 & 0,11 & 1 & 5 \\
\hline 17. Cuando me encuentro mal, me siento como si fuera una persona débil & 2,18 & 1,25 & 0,86 & $-0,33$ & 1 & 5 \\
\hline $\begin{array}{l}\text { 18. Cuando me encuentro mal, me siento culpable por sentirme de esa } \\
\text { manera }\end{array}$ & 2,00 & 1,20 & 1,05 & 0,06 & 1 & 5 \\
\hline 19. Cuando me encuentro mal, tengo dificultades para concentrarme & 2,43 & 1,23 & 0,61 & $-0,63$ & 1 & 5 \\
\hline $\begin{array}{l}\text { 20. Cuando me encuentro mal, tengo dificultades para controlar mi } \\
\text { comportamiento }\end{array}$ & 1,87 & 1,11 & 1,23 & 0,70 & 1 & 5 \\
\hline $\begin{array}{l}\text { 21. Cuando me encuentro mal, me irrito conmigo mismo por sentirme de } \\
\text { esa manera }\end{array}$ & 2,11 & 1,23 & 0,94 & $-0,19$ & 1 & 5 \\
\hline $\begin{array}{l}\text { 22. Cuando me encuentro mal, empiezo a sentirme muy mal sobre mí } \\
\text { mismo }\end{array}$ & 2,04 & 1,19 & 1,00 & 0,007 & 1 & 5 \\
\hline 23. Cuando me encuentro mal, pierdo el control sobre mi comportamiento & 1,66 & 1,04 & 1,63 & 1,88 & 1 & 5 \\
\hline $\begin{array}{l}\text { 24. Cuando me encuentro mal, tengo dificultades para pensar sobre } \\
\text { cualquier otra cosa }\end{array}$ & 2,20 & 1,18 & 0,81 & $-0,28$ & 1 & 5 \\
\hline 25. Cuando me encuentro mal, mis emociones parecen desbordarme & 1,94 & 1,19 & 1,16 & 0,33 & 1 & 5 \\
\hline
\end{tabular}

\section{Cálculo de puntuación de corte}

La DERS-E correlaciona fuerte y positivamente con OQ-45.2 $(r=0,66 ; p<0,001)$ y CES-D $(r=0,63 ; p<0,001)$.

Se calculó el área bajo la curva mediante un procedimiento no paramétrico, y se obtuvo un AUC $=0,88($ IC 95\% $=0,85-0,91)$, resultado que puede interpretarse a favor de la capacidad diagnóstica de DERS-E. Para el cálculo del punto de corte, se utilizó como criterio el valor de corte del OQ-45.2, ya que de acuerdo al cálculo de las curvas COR, permite estimar con sensibilidad y especificidad 87,9\% del área bajo la curva (Figura 1).

El nivel de concordancia entre DERS-E y el criterio de ajuste de OQ-45.2 fue moderado $(\kappa=0,421)$, con un porcentaje de coincidencia de $90,2 \%$, y se dio más en el punto de corte $=73$.

La asociación entre las variables fue esta- 


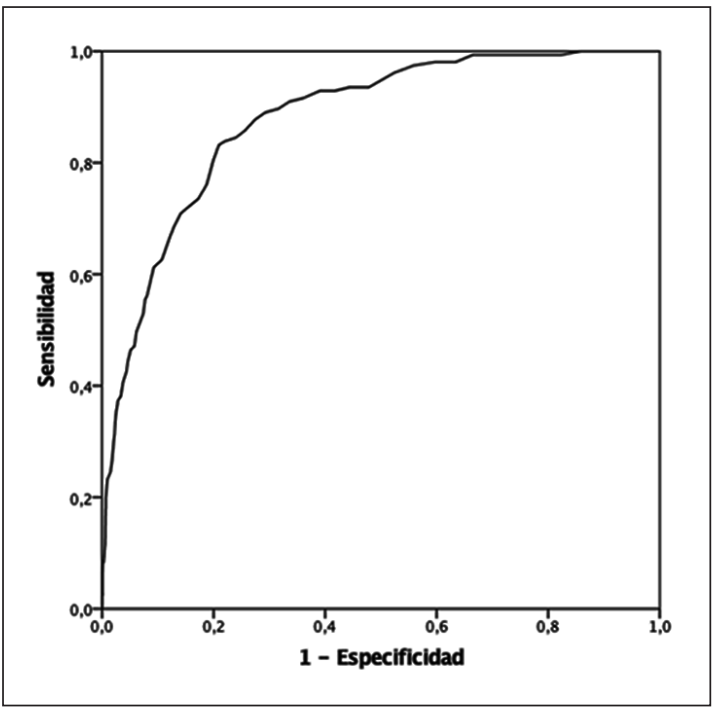

Figura 1. Curva COR.

Tabla 4. Porcentaje de personas tipificadas según puntaje de corte en las distintas muestras

\begin{tabular}{|lcc|}
\hline & $\begin{array}{c}\text { Muestra } \\
\text { clínica }\end{array}$ & $\begin{array}{c}\text { Población } \\
\text { general }\end{array}$ \\
\hline $\begin{array}{l}\text { Con dificultades de regulación } \\
\text { emocional }\end{array}$ & 42,7 & 8,4 \\
\hline $\begin{array}{l}\text { Sin dificultades de regulación } \\
\text { emocional }\end{array}$ & 57,3 & 91,6 \\
\hline
\end{tabular}

dísticamente significativa $\left(\chi^{2}=271,55 ; \mathrm{gl}=1\right.$; $p<0,01$ ), donde la probabilidad de riesgo (odd ratio) de presentar problemas de salud mental si la persona tenía o no DRE, de acuerdo a nuestro puntaje de corte, fue 8 veces mayor que quienes fueron catalogados sin DRE (OR = 8, 1 ; IC 95\% [6,13-10,63] (Tabla 4)).

\section{Discusión}

El presente estudio tuvo como objetivo elaborar normas de interpretación de las puntuaciones de DERS-E para su uso en población chilena.

Los resultados obtenidos permiten concluir que el punto de corte que permite distinguir entre personas con bajos y altos niveles de dificultades de regulación emocional es de 73 puntos, y que este es aplicable a distintos tipos de muestras: población general (incluyendo personas autodefinidas como gay o lesbiana), y que es similar para hombres y mujeres y para los distintos rangos etáreos.

La regulación emocional es un constructo que ha sido cada vez más incorporado a la investigación científica en las últimas dos décadas ${ }^{48}$, sin embargo, en Chile existe un desarrollo incipiente en este ámbito. Hasta donde tenemos conocimiento, este es el primer estudio a nivel nacional y, uno de los pocos a nivel internacional, que establece valores de referencia para las DRE. Contar con normas aplicables para la población chilena, podría ser de utilidad en dos grandes ámbitos de alta relevancia para la salud de nuestro país: el ámbito clínico y el de las políticas públicas.

En primer lugar, en el contexto clínico, las normas de referencia permiten identificar personas que tengan DRE y, por ende, mayor riesgo de presentar problemas de salud mental y física, considerando la amplia evidencia previa que documenta la asociación de las DRE con indicadores de salud y bienestar. Asimismo, disponer de estas normas permite evaluar la presencia de DRE en el inicio, desarrollo y finalización de los procesos psicoterapéuticos, contando así con indicadores de cambio concretos y confiables para la valoración del impacto de las intervenciones implementadas. También, el contar con normas abre la posibilidad de realizar comparaciones a través de distintos tipos de muestras, como, por ejemplo, con muestras clínicas y no clínicas, estudios que cotejen similitudes y diferencias entre distintos tipos de psicopatologías e, incluso, en distintas enfermedades físicas.

Por último, contar con este instrumento podría impactar las políticas públicas en salud mental en Chile, ya que podría utilizarse como herramienta de screening o tamizaje en poblaciones de alto riesgo, como es el caso, por ejemplo, de los consultantes a servicios de urgencia psiquiátrica, a centros de salud mental especializados o a programas establecidos de psicopatologías que ya han sido asociadas con DRE, como es el caso de la depresión ${ }^{12}$, problemática de alta prevalencia en nuestro país, trastornos en el consumo de sustancias $^{24}$, entre otros.

Futuras investigaciones podrían utilizar diseños longitudinales, que permitiesen seguir las trayectorias de las dificultades de regulación emocional en las personas, a través de distintos 
momentos del ciclo vital, por ejemplo, o de episodios de crisis, pudiendo de este modo evaluar el impacto de las fluctuaciones en las DRE en la activación o intensificación de psicopatologías que impactan negativamente la calidad de vida del ser humano.

\section{Referencias}

1. Salvo L, Ramírez J, Castro A. Factores de riesgo para intento de suicidio en personas con trastorno depresivo en atención secundaria. Rev Med Chile 2019; 147 (2): 181-9.

2. Ferrari A, Charlson F, Norman R, Patten S, Freedman G, Murray C, et al. Burden of depressive disorders by country, sex, age, and year: findings from the global burden of disease study 2010. PLoS Medicine 2013; 10 (11): e1001547. doi: 10.1371/journal.pmed.1001547.

3. Ministerio de Salud, Gobierno de Chile [Internet]. Santiago: División de Prevención y Control de Enfermedades; c2017-2019 [cited 2019 octubre 31]. Available from: https://diprece.minsal.cl/programas-de-salud/ salud-mental/salud-mental-en-chile/

4. Berking M, Wupperman P. Emotion regulation and mental health. Curr Opin Psychiatry 2012; 25 (2): 12834. doi:10.1097/yco.0b013e3283503669.

5. Thompson R. Emotion regulation: A theme in search of a definition. Monogr Soc Res Child Dev 1994; 59 (2-3): 25-52.

6. Gratz K, Roemer L. Multidimensional assessment of emotion regulation and dysregulation: development, factor structure, and initial validation of the difficulties in emotion regulation scale. J Psychopathol Behav Assess 2004; 26 (1): 41-54. doi: 10.1023/B:JOBA.0000007455.08539.94.

7. Lewczuk K, Kobylińska D, Marchlewska M, Krysztofiak M, Glica A, Moiseeva V. Adult attachment and health symptoms: the mediating role of emotion regulation difficulties. Curr Psychol 2018, https://doi.org/10.1007/ s12144-018-0097-z.

8. Molero O, García G. Influencia del estrés y las emociones en la hipertensión arterial esencial. Programa para el autocontrol emocional en pacientes con hipertensión arterial esencial. Rev Cubana Med 2004; 43 (2-3).

9. Silva J. Regulación emocional y psicopatología: el modelo de vulnerabilidad/resiliencia. Revista Chilena de Neuro-psiquiatría. 2005; http://dx.doi.org/10.4067/ S0717-92272005000300004

10. Porro ML, Rodríguez-Espínola S. Regulación emocional y cáncer: utilización diferencial de la expresión y supre- sión emocional en pacientes oncológicos. Avances en Psicología Latinoamericana 2012; 30 (2): 341-55.

11. Garrido-Rojas L, Guzmán-González M, Mendoza-Llanos R, Rivera D, Contreras-Garay P. Emotional regulation and depression in a sample of separated and divorced Chilean adults. Curr Psychol 2019; 1-12. https://doi. org/https://doi.org/10.1007/s12144-018-0103-5.

12. Owens G, Held P, Hamrick L, Keller E. The indirect effects of emotion regulation on the association between attachment style, depression, and meaning made among undergraduates who experienced stressful events. Motiv Emot 2018; 42: 429-37. https://doi.org/10.1007/s11031018-9688-0.

13. Özyurt G, Öztürk Y, Onat M, Mutlu C, Akay A. Attachment, emotion regulation and anger expression in adolescent depression: Did comorbid anxiety disorder not have a role? Curr Psychol 2018. https://doi.org/10.1007/ s12144-018-9985-5.

14. Stefan C, Avram J. Investigating Direct and Indirect Effects of Attachment on Internalizing and Externalizing Problems through Emotion Regulation in a Cross-Sectional Study. J Child Fam Stud 2017. DOI 10.1007/ s10826-017-0723-7.

15. Glenn C, Klonsky E. Emotion dysregulation as a core feature of Borderline Personality Disorder. J Pers Disord 2009; 23: 20-8. doi: 10.1521/pedi.2009.23.1.20.

16. Kring A, Neale J. Do schizophrenics show a disjunctive relationship among expressive, experiential, and psychophysiological components of emotion? J Abnorm Psychol 1996; 105: 249-57.

17. Roemer L, Lee JK, Salters-Pedneault K, Erisman ShM, Orsillo SM, Mennin DS. Mindfulness and emotion regulation difficulties in generalized anxiety disorder: Preliminary evidence for independent and overlapping contributions. Behav Ther 2009; 40 (2): 142-54.

18. Yap K, Mogan C, Moriarty A, Dowling N, Blair-West S, Gelgec C, Moulding R. Emotion regulation difficulties in obsessive-compulsive disorder. J Clin Psychol 2018; 74 (4): 695-709.

19. Tull M, Roemer L. Emotion regulation difficulties associated with the experience of uncued panic attacks: evidence of experiential avoidance, emotional nonacceptance, and decreased emotional clarity. Behav Ther 2007; 38: 378-91.

20. Tolin D, Levy H, Wootton B, Hallion L, Stevens M. Hoarding disorder and difficulties in emotion regulation. J Obsessive Compuls Relat Disord 2018; 16: 98 103. https://doi.org/10.1016/j.jocrd.2018.01.006.

21. Short N, Norr A, Mathes B, Oglesby M, Schmidt N. An Examination of the Specific Associations Between Facets of Difficulties in Emotion Regulation and Posttraumatic 
Stress Symptom Clusters. Cognit Ther Res 2016; 40: 783-91. DOI 10.1007/s10608-016-9787-8.

22. Gratz KL, Tull ML. The Relationship between Emotion Dysregulation and Deliberate Self-Harm Among Inpatients with Substance Use Disorders. Cognit Ther Res 2010; 34 (6): 544-53.

23. Anderson L, Claudat K, Cusack A, Brown T, Trim J, Rockwell R, Nakamura T, Gomez L, Kaye W. Differences in emotion regulation difficulties among adults and adolescents across eating disorder diagnoses. J Clin Psychol 2018; 74 (10): 1867-73.

24. Dingle G, Neves D, Alhadad S, Hides L. Individual and interpersonal emotion regulation among adults with substance use disorders and matched controls. Br J Clin Psychol 2018; 57 (2): 186-202.

25. Prosek E, Giordano A, Woehler E, Price E, McCullough R. Differences in emotion dysregulation and symptoms of depression and anxiety among illicit substance users and nonusers. Subst Use Misuse 2018; 53 (11): 1915-8.

26. Hill C, Gunderson C. Resilience of Lesbian, Gay, and Bisexual Individuals in Relation to Social Enviroment, Personal Characteristics, and Emotion Regulation Strategies. Psychol Sex Orientat Gend Divers 2015; 2 (3): 232-52. http://dx.doi.org/10.1037/sgd0000129.

27. Podder P, Mukherjee T. A study on cognition emotion regulation and locus of control in LGBT. IOSR Journal of Humanties and Social Sciences 2016; 21(12): 01-09. 10.9790/0837-2112010109.

28. Rogers A, Seager I, Haines N, Hahn H, Aldao A, Ahn $W$. Indirect effect of emotion regulation on minority stress and problematic substance use in Lesbian, Gay, and Bisexual Individuals. Front Psychol 2017; 8: 1881, doi: 10.3389/fpsyg.2017.01881.

29. Parra L, Benibgui M, Helm JL, Hastings PD. Minority Stress Predicts Depression in Lesbian, Gay, and Bisexual Emerging Adults via Elevated Diurnal Cortisol. Emerg Adulthood 2016; 4 (5): 365-72. https://doi. org/10.1177/2167696815626822.

30. Guzmán-González M, Trabucco C, Urzúa A, Garrido L, Leiva J. Validez y confiabilidad de la versión adaptada al español de la escala de dificultades de regulación emocional (DERS-E) en población chilena. Terapia Psicologica 2014; 32 (1): 19-29. https://doi.org/10.4067/ S0718-48082014000100002.

31. Cancian A, Souza L, Silva V, Machado W, Oliveira M. Psychometric properties of the brazilian versión of the Difficulties in Emotion Regulation Scale (DERS). Trends Psychiatry Psychoter 2018. DOI http://dx.doi. org/10.1590/2237-6089-2017-0128.

32. Giromini L, Ales F, Zennaro A, Pignolo C, Campora G. Developing age and gender adjusted normative refe- rence values for the Difficulties in Emotion Regulation Scale (DERS). J Psychopathol Behav Assess 2017; 39 (4): 705-14.

33. Gómez-Simón I, Penelo E, De la Osa N. Factor structure and measurement invariance of the Difficulties Emotion Regulation Scale (DERS) in Spanish adolescents. Psicothema.2014; 26 (3): 401-8.

34. Hallion L, Steinman S, Tolin D, Diefenbach G. Psychometric properties of the Difficulties in Emotion Regulation Scale (DERS) and its short forms in adults with emotional disorders. Front Psychol 2018; 9: 1-12.

35. Li J, Han Z, Gao M, Sun X, Ahemaitijiang N. Psychometric properties of the chinese version of the Difficulties in Emotion Regulation Scale (DERS): factor structure, reliability and validity. Psychol Assess 2018; 30 (5): e1e9.

36. Marín M, Robles R, González-Forteza C, Andrade P. Propiedades psicométricas de la escala "Dificultades en la Regulación Emocional” en español (DERS-E) para adolescentes mexicanos. Salud Mental 2012; 35: 521-6.

37. Medrano L, Trógolo M. Validación de la Escala de Dificultades en la Regulación Emocional en la población universitaria de Córdoba, Argentina. Universitas Psychologica 2014; 13 (4), PAG-PAG. http://dx.doi. org/10.11144/Javeriana.UPSY13-4.vedr

38. Muñoz-Martínez AM, Vargas RM, Hoyos-González JS. Escala de Dificultades en Regulación Emocional (DERS): Análisis Factorial en una Muestra Colombiana. Acta Colombiana de Psicología 2016; 19 (1): 233-44. DOI: 10.14718/ACP.2016.19.1.10.

39. Gómez-Pérez OI, Calleja-Bello N. Regulación emocional: Escalas de medición en español [revisión psicométrica]. Revista Iberoamericana de Psicología 2017; 10 (2): 183-92. Obtenido de: https://revistas.iberoamericana.edu.co/index.php/ripsicologia/article/view/1214

40. Vega P, López R. Ética en la investigación clínica. Revista Chilena de Anestesia, 2014; 43: 361-7.

41. Consejo de Organizaciones Internacionales de las Ciencias Médicas (CIOMS). Pautas éticas internacionales para la investigación relacionada con la salud con seres humanos, 2014; 136.

42. Rodríguez M. La realidad de lesbianas, gay, bisexuales, transexuales e intersexuales. Una aproximación a sus vulnerabilidades sociales. Revista Sexología y Sociedad, 2016; 22 (1): 2-14. Retrieved from http://www.medigraphic.com/pdfs/revsexsoc/rss-2016/rss161b.pdf

43. Pineda C. Etiología social del riesgo de suicidio en adolescentes y jóvenes lesbianas, gay y bisexuales: una revisión. Psicogente 2013; 16 (29): 218-34. https://doi. org/http://portal.unisimonbolivar.edu.co:82/rdigital/ psicogente/index.php/psicogente 
44. Lambert M, Burlingame G, Umphress V, Hansen N, Vermeersch D, Clouse G, et al. The reliability and validity of the Outcome Questionnaire. Clin Psychol Psychother 1996; 3: 249-58. doi: 10.1002/(SICI) 1099-0 879(199612)3:4<249::AID-CPP106>3.0.CO;2-S.

45. Von Bergen A, de la Parra G. OQ-45.2 Cuestionario para evaluación de resultados y evolución en psicoterapia: Adaptación, validación e indicaciones para su aplicación e interpretación. Terapia Psicológica 2002; 20 (2): 161-76.

46. Gempp R, Avendaño C, Muñoz C. Normas y punto de corte para la escala de depresión del Centro para Estudios Epidemiológicos (CES-D) en población juvenil chilena. Terapia Psicológica 2004; 22 (2): 145-56.

47. Cerda J, Villarroel L. Evaluación de la concordancia inter-observador en investigación pediátrica: Coeficiente de Kappa. Rev Chil Pediatr 2008; 79 (1): 54-8. https:// doi.org/10.1016/j.actatropica.2012.12.012.

48. Gómez O, Calleja N. Regulación emocional: definición, red nomológica y medición. Rev Mexicana de Investigación en Psicología 2016; 8 (1): 96-117. 